\title{
Isolation of Mesenchymal Stromal Cells (MSCs) from Human Adenoid Tissue
}

\author{
Yoon Se Lee ${ }^{a, c}$ Jae-Eun Lee ${ }^{b, c}$ Hee-Young Park ${ }^{b}$ Yun-Sung Lim ${ }^{b}$ Jin-Choon Lee ${ }^{b}$ \\ Soo-Geun Wang ${ }^{b}$ Byung-Joo Lee $^{b}$ \\ aDepartment of Otorhinolaryngology-Head and Neck Surgery, Research Institute for Convergence of \\ Biomedical Science and Technology, Pusan National University Yangsan Hospital, Yangsan, Republic \\ of Korea; ${ }^{b}$ Department of Otorhinolaryngology-Head and Neck Surgery, Pusan National University \\ Hospital and Medical Research Institute, Pusan, Republic of Korea; ${ }^{c} \mathrm{Co}$-first author
}

\section{Key Words}

Adenoid • Mesenchymal stromal cell • Differentiation • Cell surface markers

\begin{abstract}
Background: Mesenchymal stromal cells (MSCs) are multipotent progenitor cells that originally derived from bone marrow. Clinical use of bone marrow-derived MSC is difficult due to morbidity and low MSC abundance and isolation efficiency. Recently, MSCs have been isolated from various adult tissues. Here we report the isolation of adenoid tissue-derived MSCs (A-MSCs) and their characteristics. Methods: We compared the surface markers, morphologies, and differentiation and proliferation capacities of previously established tonsil-derived MSCs (T-MSCs) and bone marrow-derived MSCs (BM-MSCs) with cells isolated from adenoid tissue. The immunophenotype of A-MSCs was investigated upon interferon (IFN) $-\gamma$ stimulation. Results: A-MSCs, T-MSCs, and BM-MSCs showed negative CD45, CD31 HLA-DR, CD34, CD14, CD19 and positive CD 90, CD44, CD73, CD105 expression. A-MSCs were fibroblast-like, spindleshaped non-adherent cells, similar to T-MSCs and BM-MSCs. Adipogenesis was observed in A-MSCs by the formation of lipid droplets after Oil Red O staining. Osteogenesis was observed by the formation of the matrix mineralization in Alizarin Red staining. Chondrogenesis was observed by the accumulation of sulfated glycosaminoglycan-rich matrix in collagen type II staining. These data were similar to those of T-MSCs and BM-MSCs. Expression of marker genes (i.e., adipogenesis; lipoprotein lipase, proliferator-activator receptor-gamma, osteogenesis; osteocalcin, alkaline phasphatase, chondrogenesis; aggrecan, collagen type II $\alpha 1$ ) in A-MSCs were not different from those in T-MSCs and BM-MSCs. Conclusions: A-MSCs possess the characteristics of MSCs in terms of morphology, multipotent differentiation capacity, cell surface markers, and immunogeneity. Therefore, A-MSCs fulfill the definition of MSCs and represent an alternate source of MSCs.
\end{abstract}




\section{Introduction}

Multipotent mesenchymal stromal cells, also known as mesenchymal stromal cells (MSCs), have several distinct features from other cell types. These features are the basis of therapeutic application of MSCs. MSCs have great therapeutic potential because of their ability to self-renew and differentiate into multiple tissues. The proliferation and differentiation potential of MSCs are used in regenerative medicine [1]. In addition to their multilineage potential, MSCs have immunomodulatory properties, which are applied in bone marrow transplantation and graft-versus-host disease [2].

MSCs are fibroblast-like cells that can be isolated from a variety of tissues. MSCs were first identified in bone marrow stromal cells, and they can differentiate along multiple mesenchymal lineages, including chondrocytes, osteoblasts, and myoblasts [3, 4]. Although the bone marrow is a good source of MSCs, traditional bone marrow procurement procedures in humans are painful, frequently requiring general or spinal anesthesia, and may yield low numbers of MSCs upon processing [5]. Clinical application of MSCs requires large quantities of cells for injection or infusion. Because of the difficulty in obtaining sufficient quantities of autologous stem cells, MSCs from allogeneic donors constitute a valuable alternative source of stem cells for therapeutic applications. Therefore, alternative sources of autologous MSCs that are obtainable in large quantities with minimal discomfort have emerged, such as fat, trabecular bone, synovium, skin, thymus, periodontal ligament, palatine tonsil, placenta, and amniotic fluid [5-8].

Adenoid tissue (pharyngeal tonsil) can be obtained by adenoidectomy for adenoid vegetation, which is one of most common operations in the otolaryngologic field. Adenoid tissue is readily accessible to otolaryngologists. Although there is some evidence for palatine tonsil as a new source of MSCs [9], there are no reports on adenoid-derived MSCs (A-MSCs). Considering its histological and geographical similarities to the palatine tonsil, we hypothesized that adenoid tissue may represent an alternative source of MSCs. In this study, MSCs were isolated from adenoid tissue, and the characteristics of phenotypic A-MSCs were compared with those of tonsil-derived MSCs (T-MSCs) and bone marrow-derived MSCs (BMMSCs).

\section{Materials and Methods}

\section{A-MSC isolation and culture}

The protocol for MSC isolation from human adenoid tissue was approved by our institutional review board. Adenoid tissue was obtained after informed consent from 10 patients (age, 5-12 years old) undergoing adenoidectomy for adenoid vegetations. To isolate A-MSCs, adenoid tissues were extensively washed with equal volumes of phosphate-buffered saline (PBS), and tissues were digested at $37^{\circ} \mathrm{C}$ for 30 min with $0.075 \%$ collagenase type-I (Sigma, St. Louis, MO). Enzyme activity was neutralized with $\alpha$-modified Eagle's medium ( $\alpha$-MEM) containing 10\% fetal bovine serum (FBS), and the digested tissue was centrifuged $(1,200$ $\times \mathrm{g}, 10 \mathrm{~min}$ ) to obtain a pellet. The pellet was filtered through a $100-\mu \mathrm{m}$ nylon mesh to remove cellular debris and incubated overnight at $37^{\circ} \mathrm{C} / 5 \% \mathrm{CO}_{2}$ in control medium $(\alpha-\mathrm{MEM}, 10 \% \mathrm{FBS}, 100$ units $/ \mathrm{mL}$ of penicillin, $100 \mu \mathrm{g} / \mathrm{mL}$ of streptomycin). Following incubation, the plates were thoroughly washed with PBS to remove residual non-adherent cells. The resulting cell population was maintained at $37^{\circ} \mathrm{C} / 5 \% \mathrm{CO}_{2}$ in control medium. One week later, when the adherent-cell monolayer had reached confluency, cells were trypsinized (0.05\% trypsin-EDTA; Sigma), re-suspended in medium, and subcultured at a concentration of 2,000 cells $/ \mathrm{cm}^{2}$. The yield of non-adherent cells from each adenoid tissue ranged from $1 \times 10^{9}$ to $5 \times 10^{9}$; this morphology is indicative of a hematopoietic origin. After multiple passages with multiple buffer washes and subsequent medium changes, approximately $0.1 \%-1 \%$ of the isolated cells were found to be adherent. T-MSCs and BM-MSCs were isolated as described previously [10].

Flow cytometry analysis

Flow cytometry analysis was used to characterize the phenotypes of the A-MSCs and T-MSCs. At least 50,000 cells (in $100 \mu \mathrm{L} \mathrm{PBS}$ containing $0.5 \%$ BSA and 2 mmol/L EDTA) were incubated with FITC-conjugated 
monoclonal antibodies against human CD45, CD31, HLA-DR, CD34, CD14, CD19 and for CD90, CD44, CD73, CD105 (BD Biosciences Clontech, Palo Alto, CA) or with the respective isotype control controls. Labeled cells were analyzed by flow cytometry using a FACS Caliber flow cytometer and the Cell Quest Pro software (BD Biosciences, San Diego, CA).

Colony numbers per adherent cell.

Nucleated cells from adenoids and tonsils were plated at $1 \times 10^{5}, 1 \times 10^{6}$, and $1 \times 10^{7}$ cells $/ 60-\mathrm{cm}^{2}$ dishes and cultured for 7 days. The dishes were stained with $0.5 \%$ crystal violet and the colonies were counted. Colonies less than $2 \mathrm{~mm}$ in diameter and faintly stained colonies were ignored.

\section{Colony-forming assays}

At the second passage, 100 cells were plated and cultured for 7 days in $60-\mathrm{cm}^{2}$ dishes. Cells were subsequently fixed with $4 \%$ paraformaldehyde, stained with $0.5 \%$ crystal violet in $4 \%$ paraformaldehyde for $5 \mathrm{~min}$, and washed twice with distilled water. The number of colonies was then counted.

\section{Multilineage differentiation}

The capacities of A-MSCs, T-MSCs and BM-MSCs to differentiate into the adipogenic, osteogenic, and chondrogenic lineages were analyzed. Adipogenic differentiation was induced by culturing T-MSCs for 3 weeks in adipogenic medium $(1 \mu \mathrm{M}$ dexamethasone [Sigma], $100 \mu \mathrm{g} / \mathrm{mL}$ 3-isobutyl-1 methylxanthine (IBMX), $5 \mu \mathrm{g} / \mathrm{mL}$ insulin, $60 \mu \mathrm{M}$ indomethacine, and 10\% FBS in $\alpha$-MEM) and assessed using Oil Red 0 (Sigma) staining as an indicator of intracellular lipid accumulation. Prior to staining, cells were fixed in $70 \%$ ethanol for $15 \mathrm{~min}$ at room temperature and then incubated in 2\% Oil Red 0 reagent for $1 \mathrm{~h}$ at room temperature. Excess stain was removed by washing with distilled water.

Osteogenic differentiation was induced by culturingMSCs for 3 weeks in osteogenic medium $(0.1 \mathrm{mM}$ dexamethasone, $10 \mu \mathrm{M} \beta$-glycerophosphate, $50 \mu \mathrm{g} / \mathrm{mL}$ ascorbic acid, and 10\% FBS in $\alpha$-MEM). Extracellular matrix calcification was examined by Alizarin Red S (Sigma). Briefly, cells were fixed with 70\% ethanol, washed with distilled water, and incubated in $2 \%$ Alizarin Red S for $15 \mathrm{~min}$ at room temperature, followed by multiple washes with distilled water.

Chondrogenic differentiation was induced using the micromass culture technique. Briefly, $10 \mu \mathrm{L}$ of concentrated MSC suspension $\left(3 \times 10^{5}\right.$ cells $\left./ \mathrm{mL}\right)$ was plated in the center of each well and allowed to attach at $37^{\circ} \mathrm{C}$ for $2 \mathrm{~h}$. Chondrogenic medium (CM; $1 \%$ FBS, $0.1 \mathrm{mM}$ dexamethasone, $50 \mu \mathrm{g} / \mathrm{mL}$ ascorbic acid, ITS +1 [insulin-transferrin-selenium; Sigma], $10 \mathrm{ng} / \mathrm{mL}$ TGF- $\beta 1$ [Sigma], and $10 \mathrm{ng} / \mathrm{mL}$ in $\alpha-M E M$ ) was gently overlaid so as not to detach the cell nodules, and the culture was maintained in CM for 4 weeks before analysis. Chondrogenesis was confirmed by immunohistochemistry for collagen type-II. Briefly, sections were blocked with $10 \%$ horse serum, incubated with purified anti-mouse collagen type-II antibody (BD Biosciences, San Jose, CA) for $1 \mathrm{~h}$, and washed with PBS (pH 7.4). Antibody-bound cells were detected with a peroxidase substrate kit (Vectastain ABC kit; Vector Laboratories, Burlingame, CA). Sections were washed, counterstained with hematoxylin, and examined by light microscopy.

Real-time quantitative reverse transcription-polymerase chain reaction (PCR) for gene expression

Total RNA was isolated from MSCs from day 21 monolayers and pellet cultures, using the TRIzol reagent or method (Invitrogen, Carlsbad, CA). The isolated RNA was then reverse-transcribed using random hexamers. Real-time PCRs were performed using 10 ng of cDNA and SYBR Green mix (Bio-Rad Laboratories, Hercules, CA). Gene-specific primers were designed based on GenBank cDNA sequences, as described previously (Table 1) [9]. Lipoprotein lipase (LPL) and peroxisome proliferator-activated receptor-gamma (PPAR $\gamma$ ) were used as genetic markers of adipogenesis, alkaline phosphatase (ALP) and osteocalcin (OC) for osteogenesis, and collagen type-II $\alpha 1$ (COL2A1) and aggrecan (AGN) for chondrogenesis. Expression levels are presented as fold-increases over glyceraldehyde-3-phosphate dehydrogenase (GAPDH) expression, using the formula $2^{(\Delta \mathrm{Ct})}$, where $\Delta \mathrm{Ct}=\mathrm{Ct}$ of target gene $-\mathrm{Ct}$ of house-keeping gene (GAPDH).

\section{Statistical analyses}

Statistical analyses were performed using the SPSS software (ver. 15.0; Chicago, IL). Differences between the levels of gene expression were evaluated using the Krusal-Wallis test. A $p$-value $<0.05$ was considered significant. 
Lee et al.: Alternate Source of Mesenchymal Stromal Cells

\begin{tabular}{|c|c|c|}
\hline Genes & Primer sequences $\left(5^{\prime}-3^{\prime}\right)$ & Predicted size (bp) \\
\hline GADPH & $\begin{array}{l}\text { GGACTCATGACCACAGTCCATGCC } \\
\text { TCAGGGATGACCTTGCCCACA }\end{array}$ & 152 \\
\hline$A L P$ & $\begin{array}{l}\text { TGGAGCTTCAGAAGCTCAACACCA } \\
\text { ATCTCGTTGTCTGAGTACCAGTCC }\end{array}$ & 454 \\
\hline$O C$ & $\begin{array}{l}\text { ATGAGAGCCCTCACACTCCTC } \\
\text { GCCGTAGAAGCGCCGATAGGC }\end{array}$ & 294 \\
\hline$L P L$ & $\begin{array}{l}\text { GAGATTTCTCTGTATGGCACC } \\
\text { CTGCAAATGAGACACTTTCTC }\end{array}$ & 276 \\
\hline PPARY & $\begin{array}{l}\text { TGAATGTGAAGCCCATTGAA } \\
\text { CTGCAGTAGCTGCACGTGTT }\end{array}$ & 161 \\
\hline$A G N$ & $\begin{array}{l}\text { TGCGGGTCAACAGTGCCTATC } \\
\text { CACGATGCCTTTCACCACGAC }\end{array}$ & 182 \\
\hline COL2A1 & $\begin{array}{l}\text { GGAAACTTTGCTGCCCAGATG } \\
\text { TCACCAGGTTCACCAGGATTGC }\end{array}$ & 167 \\
\hline
\end{tabular}

Table 1. Primers used in reverse transcriptionpolymerase chain reaction

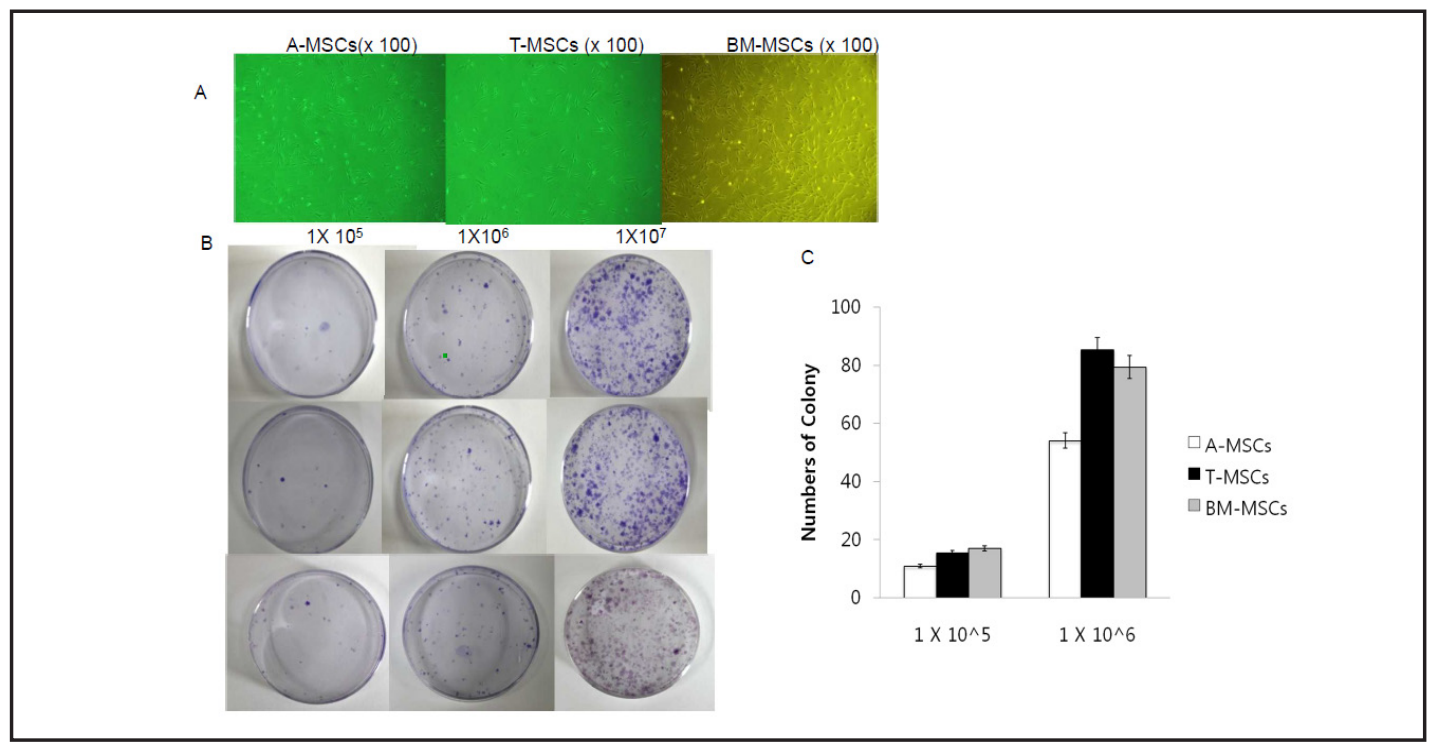

Fig. 1. (A) Morphology of adenoid-derived mesenchymal stromal cells (A-MSCs, right), tonsil-derived mesenchymal stromal cells (T-MSCs, middle), and bone marrow-derived stromal cells (BM-MSCs, left). (B) Colony forming units (CFU) after passage 2 at 7 days (upper row; T-MSCs, middle row; A-MSCs, lower row; BM-MSCs). (C) Numbers of CFU after culture of $1 \times 10^{5}$ and $1 \times 10^{6}$ cells of MSCs at passage 0 , respectively. Five individual experiments were performed $(*, p<0.05)$.

\section{Results}

Cell viability, proliferation, and clonogenecity

We harvested 20 tonsillectomy and 10 adenoidectomy specimens to isolate MSCs. The mean age of the palatine tonsil donors $(20.7 \pm 14.9$ years) was higher than that of the adenoid donors $(10.2 \pm 7.6$ years; $p<0.05)$. The palatine tonsil donors included 12 women and 8 men, and adenoid donors included 5 women and 5 men.

To compare the morphology of A-MSCs with T-MSCs and BM-MSCs, we examined cells freshly isolated from the adenoid after adenoid specimen processing. Three different cell morphologies were observed: (a) fibroblast-like spindle-shaped morphology, (b) round morphology with large nuclei (monocytic contamination), and (c) very small, epithelioid cells with polygonal morphology. After repeated trypsinization, only cells with the fibroblast-like spindle-shaped morphology were remained. The morphology of A-MSCs was similar to that of T-MSCs (Fig. 1A). At passage 2, the colony-forming units (CFUs) of A-MSCs, T-MSCs, and BM-MSCs were numerated following inoculation with different numbers of cells to evaluate the proliferation potential of the MSCs at day 7 (Fig. 1B). 
Fig. 2. Representative flow cytometry graphs and mean value ( \pm standard error) of cell surface epitope. (A) CD90, CD44, CD73, and CD105 were used as positive markers. (B) CD45, CD31, HLA-DR, CD34, CD14, and CD19 were used as negative markers. Three individual experiments were performed.

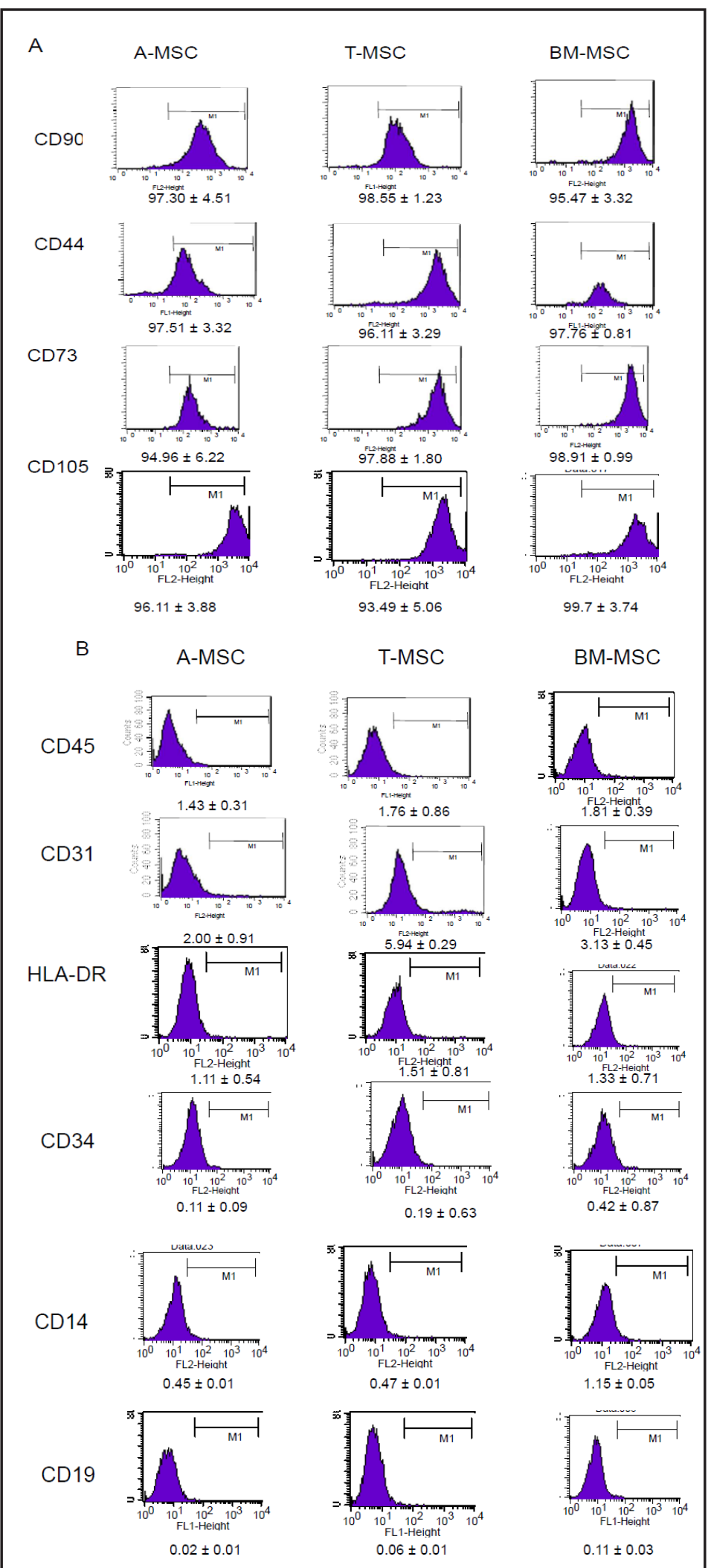

T-MSC

$1.76 \pm 0.86$
$98.91 \pm 0.99$

BM-MSC

$3.13 \pm 0.4$

Upon plating at a limiting dilution under colony-forming unit-fibroblasts (CFU-F) assay conditions, the T-MSC isolates exhibited similar changes in colony and cell numbers, suggesting that the A-MSCs were limiting for CFU-F (Fig. 1C). The numbers of CFUs of both 


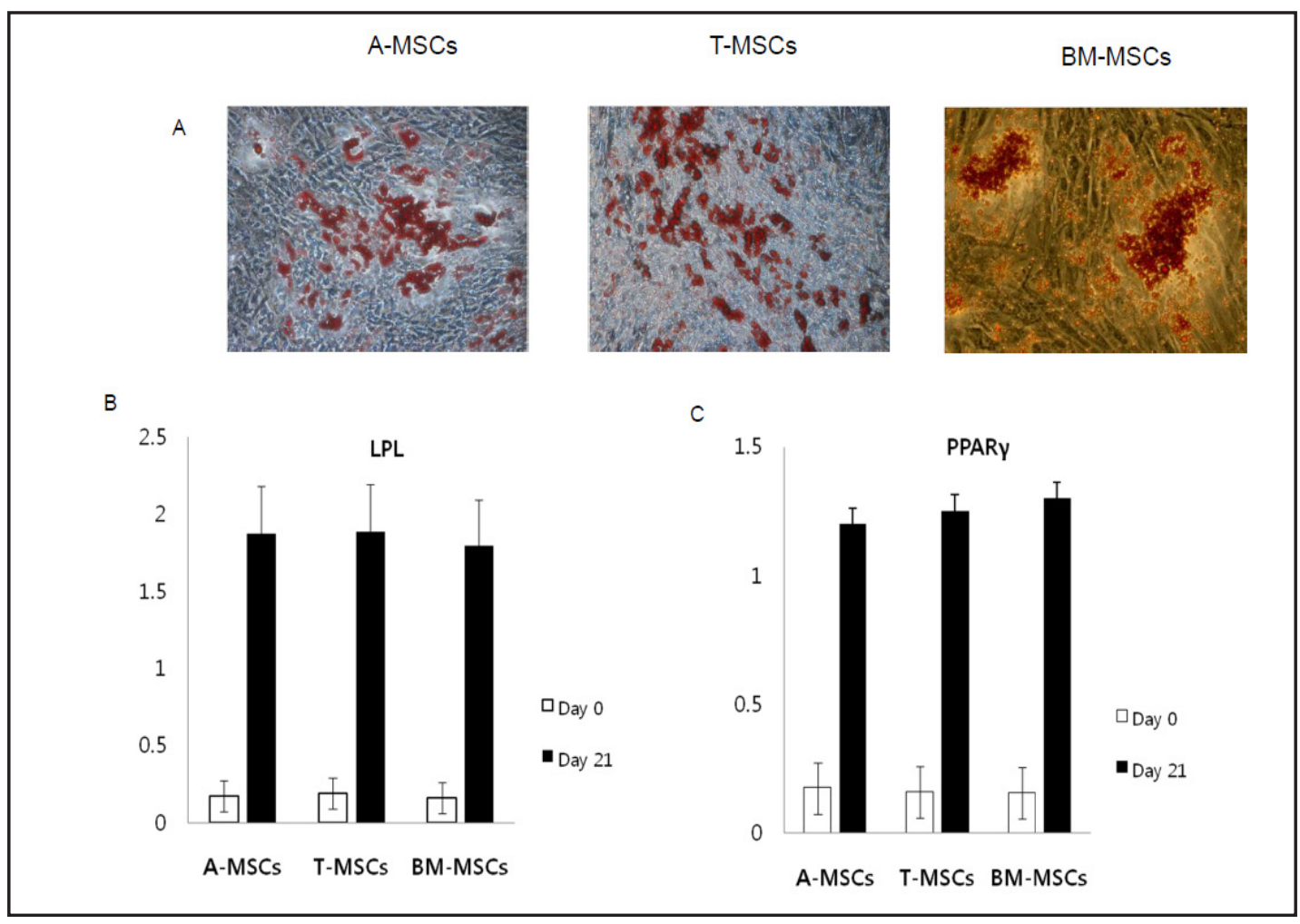

Fig. 3. Adipogenic differentiation potential of MSCs. (A) Lipid droplet were stained with Oil Red O. (left column; A-MSCs, middle column; T-MSCs, right column; BM-MSCs). Real-time reverse transcription polymerase chain reaction (RT-PCR) analysis of adipogenic differentiation markers, lipoprotein lipase $(L P L)(B)$ and proliferator-activated receptor-gamma (PPAR $\gamma$ ) (C) was performed in A-MSCs and T-MSCs on day 0 and 21. The vertical axis of the graph shows relative gene expression level. Gene expression levels are presented as relative expression over glyceraldehydes 3-phosphate dehydregenase (GAPDH). Specific transcript levels of marker genes were normalized by comparison to those of GAPDH.

MSCs increased with an increase in the inoculated cell number. When $1 \times 10^{6}$ cells were cultured, A-MSCs $(54.2 \pm 21.12)$ exhibited fewer CFUs than T-MSCs or BM-MSCs $(85.4 \pm$ 42.24 and $79.4 \pm 31.11$, respectively) $(p<0.001)$. The CFU-F frequency in the adenoid digest was approximately 1 in 6,000 cells plated.

\section{Cell surface markers}

Human MSCs are difficult to define given the lack of a specific marker. Cell surface epitopes were analyzed using flow cytometry for the minimal criteria in addition to morphology and proliferation potential [11]. A-MSCs exhibited a cell surface epitope profile similar to that of T-MSCs and BM-MSCs. The A-MSCs, T-MSCs, and BM-MSCs exhibited positive CD 90, CD44, CD73, and CD105 expression in a similar pattern (Fig. 2). Negative expression of CD45 and CD31 in both the MSCs indicates that these MSCs are not either hematopoietic cells and nonendothelial cells. HLA-DR negativity in both MSCs suggests a lack of immunogenicity. CD34, CD14, and CD19 were also negative in these MSCs.

Multileneage differentiation potential toward adipogenesis, osteogenesis and chondrogenesis

Both MSCs were treated with adipogenic supplements and cultured in expansion medium after passage 2. Morphologically, the formation of cytoplasmic lipid droplets was noticed after 1 week of adipogenic induction, as indicated by Oil Red 0 staining (Fig. 3A). Expression of $L P L$ and PPAR $\gamma$ mRNA, which are markers of adipogenic differentiation, 


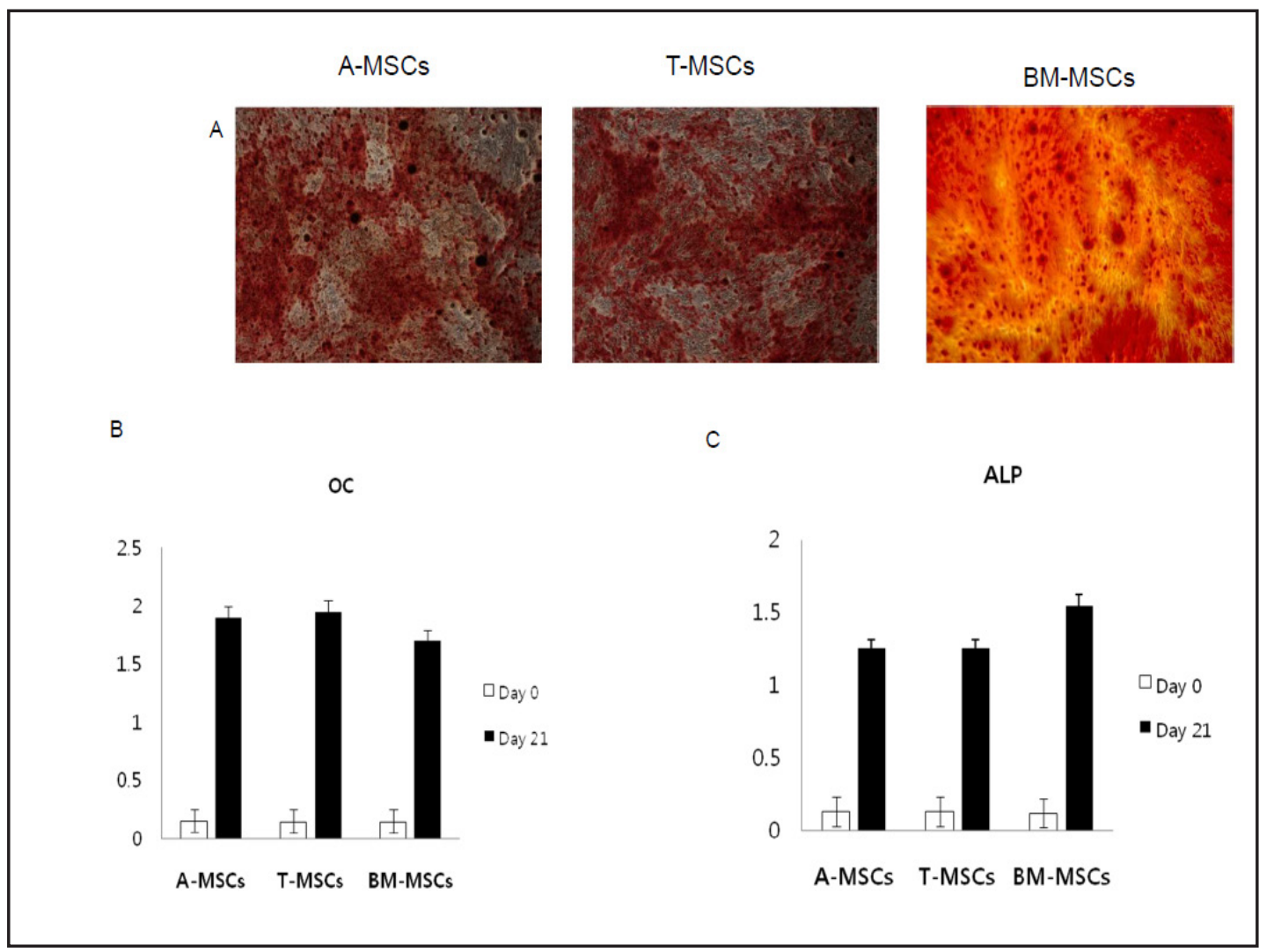

Fig. 4. Osteogenic differentiation potential of MSCs. (A) The mineralized matrix was stained with Alizarin Red S. (B, C) Real-time RT-PCR analysis of osteogenic differentiation markers, osteocalcin $(O C)$ and alkaline phosphatase $(A L P)$ was performed in A-MSCs, T-MSCs, and BM-MSCs on day 0 and 21. The vertical axis of the graph shows relative gene expression level. Gene expression levels are presented as relative expression over glyceraldehydes 3-phosphate dehydregenase (GAPDH). Specific transcript levels of marker genes were normalized by comparison to those of GAPDH.

were enhanced after 21 days of induction on both A-MSCs and T-MSCs (Fig. 3B, 3C). The expression level of $L P L$ in BM-MSCs $(1.79 \pm 0.05)$ was lower than in A-MSCs $(1.88 \pm 0.06)$ or T-MSCs (1.89 \pm 0.05$)$ at day 21, while the level of PPAR $\gamma$ in A-MSCs $(1.20 \pm 0.03)$ were lower than in T-MSCs $(1.25 \pm 0.05)$ or BM-MSCs $(1.30 \pm 0.03)$ at day 21 . These differences were not significant.

The morphology of both A-MSCs and T-MSCs was flattened following osteogenic induction by osteogenic supplements and culture in expansion medium. Differentiation into osteoblasts was confirmed by positive Alizarin Red S staining (Fig. 4A). Expression levels of $O C$ mRNA were increased in both MSCs (Fig. 4B, 4C). The expression level of $O C$ in BM-MSCs $(1.74 \pm 0.06)$ was lower than in A-MSCs $(1.90 \pm 0.05)$ or T-MSCs $(1.95 \pm 0.06)$ at day 21 , while the level of $A L P$ in BM-MSCs $(1.54 \pm 0.03)$ were higher than in A-MSCs $(1.25 \pm 0.7)$ or T-MSCs $(1.25 \pm 0.05)$ at day 21 . These differences were not significant.

The chondrogenic differentiation potential of both MSCs was assessed using highdensity pellet cultures, which were maintained in chondrogenic medium. After 3 weeks of culture, matrix-sulfated proteoglycan accumulation in chondrogenic cultures was detectable by immunohistochemical collagen type-II staining (Fig. 5A). Real-time RT-PCR analysis revealed a significant increase in AGN and COL2 expression in both A-MSCs and T-MSCs (Fig. 5B, 5C). Compared with that of naïve MSCs, relative expression levels of $A G N(3.03 \pm 0.15)$ and COL2 $(3.63 \pm 0.13)$ in A-MSCs at day 21 were not significantly different from those in 


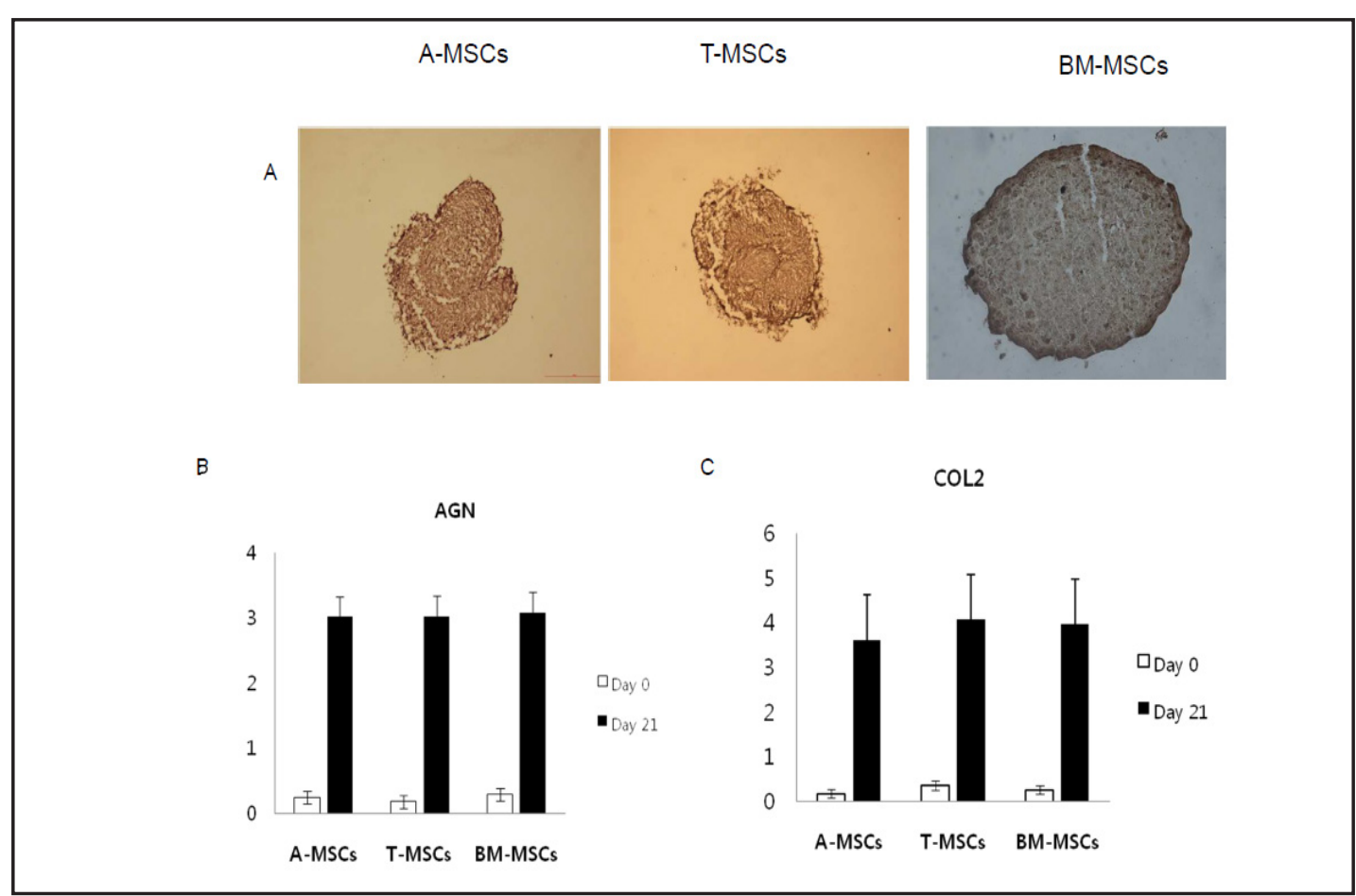

Fig. 5. Chondrogenic differentiation potential of MSCs. (A) Sulfated glycosaminoglycan-rich matrix was stained with immunohistochemistry for collagen type-II $\alpha 1$. Real-time RT-PCR analysis of chondrogenic differentiation markers, aggrecan ( $A G N$ ) and collagen type-II $\alpha 1$ (COL2A1) was performed in A-MSC, T-MSCs, and BM-MSCs on day 0 and 21. The vertical axis of the graph shows relative gene expression level. Gene expression levels are presented as relative expression over glyceraldehydes 3-phosphate dehydregenase (GAPDH). Specific transcript levels of marker genes were normalized by comparison to those of GAPDH.

Fig. 6. Expression level of HLADR was evaluated by flow cytometry in A-MSCs for 2 days. (A) The cell surface expression of unstimulated A-MSCs is regarded as control. (B) After treatment of cells with IFN- $\gamma$, expression of HLA-DR is compared to control. Similar results were obtained with A-MSCs from three individual experiments $(p<0.05)$.

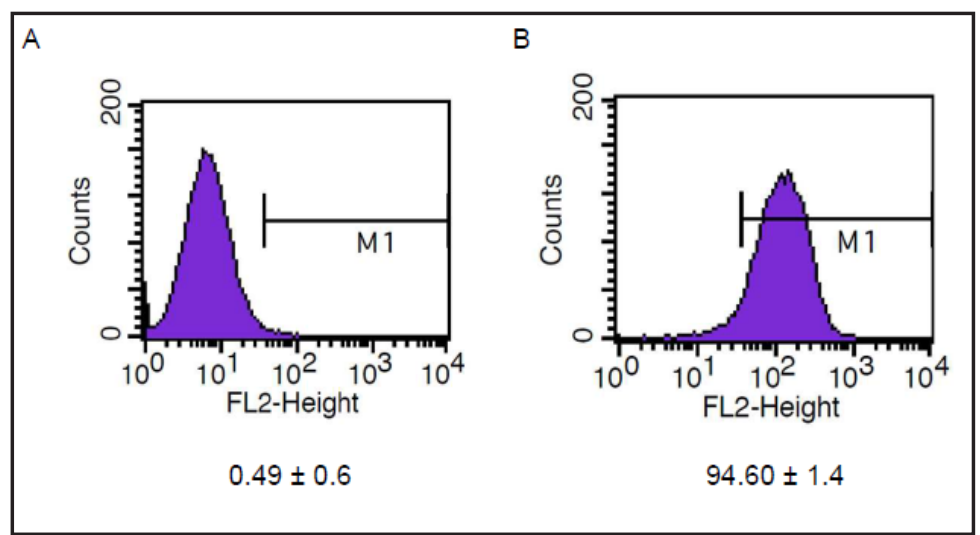

T-MSCs (AGN; $3.04 \pm 0.16$, COL2; $4.09 \pm 0.23)$ or BM-MSCs $(A G N ; 3.1 \pm 0.27$, COL2; $4.09 \pm$ $0.23)$.

\section{HLA-DR change after IFN- $\gamma$ stimulation}

Unstimulated A-MSCs was negative for HLA-DR (0.35\%, Fig. 6A). To reveal the level change of HLA-DR in A-MSCs upon immunological stimulation, we treated A-MSCs with 200IU of recombinant human IFN- $\gamma$ for 2 days. The expression of HLA-DR on A-MSCs following stimulation (94.6\%) was markedly increased compared with that of unstimulated A-MSCs (Fig. 6B). Similar results were obtained with A-MSCs from two individuals. 


\section{Discussion}

Tissue-derived MSCs are distributed in almost all tissues, such as bone marrow, muscle, fat, hair follicle, tooth root, placenta, brain, periosteum, dermis, perichondrium, umbilical cord and so on [12]. The stroma of adenoid tissue is composed of lymphoid and non-lymphoid cells. Primordial gland in the posterior nasopharynx, where pharyngeal crypts are formed, is infiltrated with lymphoid tissue and the mucosa is covered with pseudostratified ciliated epithelia during development [13]. This embryological concept led us to hypothesize that MSCs can be isolated from adenoid tissue.

MSCs are typically defined by a combination of phenotypic and functional characteristics. MSCs are non-hematopoietic cells that possess multi-lineage differentiation potential and a high capacity for self-replication [5]. To the best of our knowledge, this is the first report of MSC isolation form the adenoid tissue. In this study, fibroblast-like cells isolated from the adenoid were similar to T-MSCs [10]. The proliferation potential of A-MSCs was indicated by the increasing CFUs after multiple passages. This implies that sufficient A-MSCs can be isolated for clinical purposes. The three MSCs may exhibit minor differences in proliferation rate, which was not evaluated here [14].

Next, we investigated whether the A-MSCs fulfilled the minimum requirement for MSCs in terms of cell surface markers, differentiation potential, and immunological phenotypes [11]. The epitope profiles of both MSCs were characterized by flow cytometry, and neither expressed the hematopoietic marker CD45, CD34, and CD14 or the endothlelial cell marker CD31, whereas the MSC-positive markers CD73, CD90, and CD105 were detected $[5,15]$. The lack of immunogenicity in A-MSCs was supported by their HLA-DR negativity.

Multilineage differentiation potential was demonstrated by the generation of adipocytes, osteoblasts, and chondrocytes from A-MSCs and T-MSCs. Adipocytes displayed characteristic lipid droplets and expressed LPL and PPAR mRNAs. Analysis of cells growing under osteogenic conditions revealed areas of mineralization and osteoblasts. $O C$ and ALP mRNAs were also expressed. Chondrocytes displayed accumulation of sulfated glycosaminoglycans in cell pellets and expressed AGN and COL2 mRNAs. Importantly, the levels of various mRNAs in each differentiated cell type did not differ significantly between A-MSCs, T-MSCs and BM-MSCs. Autologous and allogeneic MSCs are being used in various fields from tissue regeneration to reconstruction $[16,17]$. Considering immunological tissue rejection, autologous tissue is an ideal source of MSCs. However, local or general anesthesia to obtain tissues as a source of MSCs, inevitable delays in injection due to culture period, and poor general condition may be some limitations to the use of autologous MSCs. Repetitive procedures or longer culture period to obtain adequate quantities of MSCs may be needed in some patients. Thus, immune reaction-free allogeneic MSCs are considered as an alternative material for use in regeneration or reconstruction.

MSCs are less immunogenic and secrete several immunosuppressive factors. MSCs suppress the activation of T- and B-lymphocytes as well as natural killer (NK) cells and dendritic cells [18]. Because certain co-stimulatory molecules such as CD86, CD40, or CD40L are not detectable, MSCs may not be recognized by alloreactive T- cells and are not rejected when injected in allogeneic recipients [5]. Membrane molecules that activate immune cells (e.g., HLA-DR) are expressed at low levels in MSCs. Bone marrow- or adipose tissue-derived MSCs exhibit immunomodulatory functions that are mediated by secretory molecules, including TGF- $\beta 1$, hepatocyte growth factor, prostaglandin E2, indoleamine 2,3-dioxygenage, and HLA-G5 $[18,19]$. This characteristic is used in the treatment of immune or immunerelated diseases, such as graft-versus-host disease. The infusion of MHC-mismatched MSCs, both in animals and humans, is well tolerated and clinically efficacious [20,21]. Recently, T-MSCs were shown to have immunomodulatory functions similar to those of previously described BM-MSCs [22]. Although immunomodulatory functions of A-MSCs were not evaluated in this study, low levels of HLA-DR may hint such a function. In this regard, the immunomodulatory effect of MSCs enables the use of these cells in allogeneic conditions [23]. 
Lower donor morbidity and accessibility are prerequisites for immunologically safe allogeneic stem cells. Tissues from young donors exhibit higher proliferation potential than those from older donors. Adenoidectomy is usually performed in patients below 10 years of age. The cellular proliferative capacity and multilineage differentiation potential of MSCs are influenced by the age of the donors from which the MSCs were harvested [24, 25]. The adenoid tissue from which A-MSCs were isolated (by adenoidectomy) was usually obtained from young patients suffering from adenoid vegetation, which may ameliorate the age-related cell yield problem. Therefore, A-MSCs would not be affected on age-related dysfunctions of MSCs. In addition, tonsillectomy and adenoidectomy are the most familiar operations performed by otolaryngologists. A substantial amount of tissue can be harvested during these operations, which may preclude the need for additional procedures. Adenoid tissue from adenoidectomies can be stored and used later for autologous or allogeneic transplantation, in the same way that as cord blood is stored for future purpose. In this regard, allogeneic MSCs isolated from the adenoid tissue have a benefit of large yield of MSCs without allogeneic reaction. Furthermore, their pluripotential differentiation ability is used in regenerative medicine $[26,27]$. A-MSCs can be applied for allogeneic transplantation to regenerate scar tissue is the same way that other tissue-originated MSCs have been used; MSCs are also being tested as a therapeutic for otolaryngologic diseases such as hearing loss, facial nerve deficit, radiation-induced xerostomia, and vocal cord scar [28-32].

\section{Conclusion}

MSCs are widely used in the treatment of immune-related diseases and in regenerative medicine. In this study, we isolated MSCs from adenoid tissue and found that the morphology, cell surface markers, proliferation potential and differentiation potential of A-MSCs were similar to those of T-MSCs and BM-MSCs. Thus, adenoid tissue represents a good alternative source of MSCs, and is familiar to otolaryngologists. This study may be a basis for the clinical application of A-MSCs in regenerative and immune-related diseases. Further studies are needed to optimize the cell population and application of A-MSCs as an alternative source of allogeneic MSCs.

\section{Declaration of interest}

The authors report no conflicts of interest. The authors alone are responsible for the content and writing of the paper.

\section{Abbreviations}

GAPDH (glyceraldehyde-3-phosphate dehydrogenase); LPL (lipoprotein lipase); PPAR $\gamma$ (proliferator-activated receptor-gamma); OC (osteocalcin); ALP (alkaline phosphatase); AGN (aggrecan); COL2A1 (collagen type-II $\alpha 1$ ).

\section{Acknowledgements}

This study was supported by the Grant (2008-10) from Pusan National University Researche Institute. 
Lee et al.: Alternate Source of Mesenchymal Stromal Cells

\section{References}

1 Caplan AI: Adult mesenchymal stem cells for tissue engineering versus regenerative medicine. J Cell Physiol 2007;213:341-347.

-2 Le Blanc K, Rasmusson I, Sundberg B, Gotherstrom C, Hassan M, Uzunel M, Ringden O: Treatment of severe acute graft-versus-host disease with third party haploidentical mesenchymal stem cells. Lancet 2004;363:1439-1441.

3 Kolf CM, Cho E, Tuan RS: Mesenchymal stromal cells. Biology of adult mesenchymal stem cells: Regulation of niche, self-renewal and differentiation. Arthritis Res Ther 2007;9:204.

$\checkmark 4$ Kuo CK, Li WJ, Mauck RL, Tuan RS: Cartilage tissue engineering: Its potential and uses. Curr Opin Rheumatol 2006;18:64-73.

-5 Pittenger MF, Mackay AM, Beck SC, Jaiswal RK, Douglas R, Mosca JD, Moorman MA, Simonetti DW, Craig S, Marshak DR: Multilineage potential of adult human mesenchymal stem cells. Science 1999;284:143-147.

-6 In 't Anker PS, Scherjon SA, Kleijburg-van der Keur C, de Groot-Swings GM, Claas FH, Fibbe WE, Kanhai HH: Isolation of mesenchymal stem cells of fetal or maternal origin from human placenta. Stem Cells 2004;22:1338-1345.

7 In 't Anker PS, Scherjon SA, Kleijburg-van der Keur C, Noort WA, Claas FH, Willemze R, Fibbe WE, Kanhai HH: Amniotic fluid as a novel source of mesenchymal stem cells for therapeutic transplantation. Blood 2003;102:1548-1549.

8 Zuk PA, Zhu M, Mizuno H, Huang J, Futrell JW, Katz AJ, Benhaim P, Lorenz HP, Hedrick MH: Multilineage cells from human adipose tissue: Implications for cell-based therapies. Tissue Eng 2001;7:211-228.

-9 Janjanin S, Djouad F, Shanti RM, Baksh D, Gollapudi K, Prgomet D, Rackwitz L, Joshi AS, Tuan RS: Human palatine tonsil: A new potential tissue source of multipotent mesenchymal progenitor cells. Arthritis Res Ther 2008;10:R83.

10 Lee RH, Kim B, Choi I, Kim H, Choi HS, Suh K, Bae YC, Jung JS: Characterization and expression analysis of mesenchymal stem cells from human bone marrow and adipose tissue. Cell Physiol Biochem 2004;14:311324.

11 Dominici M, Le Blanc K, Mueller I, Slaper-Cortenbach I, Marini F, Krause D, Deans R, Keating A, Prockop Dj, Horwitz E: Minimal criteria for defining multipotent mesenchymal stromal cells. The international society for cellular therapy position statement. Cytotherapy 2006;8:315-317.

12 Bianco P, Robey PG, Simmons PJ: Mesenchymal stem cells: Revisiting history, concepts, and assays. Cell Stem Cell 2008;2:313-319.

13 Ali MY: Histology of the human nasopharyngeal mucosa. J Anat 1965;99:657-672.

14 Baxter MA, Wynn RF, Jowitt SN, Wraith JE, Fairbairn LJ, Bellantuono I: Study of telomere length reveals rapid aging of human marrow stromal cells following in vitro expansion. Stem Cells 2004;22:675-682.

15 Prockop DJ: Marrow stromal cells as stem cells for nonhematopoietic tissues. Science 1997;276:71-74.

16 Pittenger MF, Martin BJ: Mesenchymal stem cells and their potential as cardiac therapeutics. Circ Res 2004;95:9-20.

17 Schafer R, Dominici M, Muller I, Dazzi F, Bieback K, Godthardt K, Le Blanc K, Meisel R, Pochampally R, Richter R, Skutella T, Steinhoff G, Mitterberger M, Wendel H, Wiskirchen J, Handgretinger R, Northoff H: Progress in characterization, preparation and clinical applications of non-hematopoietic stem cells, 29-30 September 2006, Tubingen, Germany. Cytotherapy 2007;9:397-405.

18 Selmani Z, Naji A, Gaiffe E, Obert L, Tiberghien P, Rouas-Freiss N, Carosella ED, Deschaseaux F: Hla-g is a crucial immunosuppressive molecule secreted by adult human mesenchymal stem cells. Transplantation 2009;87:S62-66.

19 Djouad F, Plence P, Bony C, Tropel P, Apparailly F, Sany J, Noel D, Jorgensen C: Immunosuppressive effect of mesenchymal stem cells favors tumor growth in allogeneic animals. Blood 2003;102:3837-3844.

20 Devine SM, Bartholomew AM, Mahmud N, Nelson M, Patil S, Hardy W, Sturgeon C, Hewett T, Chung T, Stock W, Sher D, Weissman S, Ferrer K, Mosca J, Deans R, Moseley A, Hoffman R: Mesenchymal stem cells are capable of homing to the bone marrow of non-human primates following systemic infusion. Exp Hematol 2001;29:244-255.

-21 Spaggiari GM, Capobianco A, Becchetti S, Mingari MC, Moretta L: Mesenchymal stem cell-natural killer cell interactions: Evidence that activated nk cells are capable of killing mscs, whereas mscs can inhibit il-2induced nk-cell proliferation. Blood 2006;107:1484-1490. 
22 Lee YS PH, Lim YS, Lee J, Wang S, Jung JS, Lee B: Can the palatine tonsil be a source of mesenchymal stem cells with immunomodulatory property? J Clin Otolaryngol 2012;23:1-11.

23 Aggarwal S, Pittenger MF: Human mesenchymal stem cells modulate allogeneic immune cell responses. Blood 2005;105:1815-1822.

24 Bajek A, Czerwinski M, Olkowska J, Gurtowska N, Kloskowski T, Drewa T: Does the mesenchymal stem cells aging limit their potential application in clinical practice? Aging Clin Exp Res 2012;24:404-411.

-25 Zheng H, Martin JA, Duwayri Y, Falcon G, Buckwalter JA: Impact of aging on rat bone marrow-derived stem cell chondrogenesis. J Gerontol A Biol Sci Med Sci 2007;62:136-148.

-26 Jones BA, Pei M: Synovium-derived stem cells: A tissue-specific stem cell for cartilage engineering and regeneration. Tissue Eng Part B Rev 2012;18:301-311.

27 Munoz Ruiz M, Regueiro JR: New tools in regenerative medicine: Gene therapy. Adv Exp Med Biol 2012;741:254-275.

28 Caylan R, Bektas D, Dikmen T, Bektas O, Omay SB, Ovali E: Mesenchymal stem cells in iatrogenic facial nerve paralysis: A possible role in the future. Eur Arch Otorhinolaryngol 2006;263:963-967.

29 Cho KS, Park HK, Park HY, Jung JS, Jeon SG, Kim YK, Roh HJ: Ifats collection: Immunomodulatory effects of adipose tissue-derived stem cells in an allergic rhinitis mouse model. Stem Cells 2009;27:259-265.

-30 Ito J, Murata M, Kawaguchi S: Regeneration and recovery of the hearing function of the central auditory pathway by transplants of embryonic brain tissue in adult rats. Exp Neurol 2001;169:30-35.

-31 Lee BJ, Wang SG, Lee JC, Jung JS, Bae YC, Jeong HJ, Kim HW, Lorenz RR: The prevention of vocal fold scarring using autologous adipose tissue-derived stromal cells. Cells Tissues Organs 2006;184:198-204.

-32 Lombaert IM, Wierenga PK, Kok T, Kampinga HH, deHaan G, Coppes RP: Mobilization of bone marrow stem cells by granulocyte colony-stimulating factor ameliorates radiation-induced damage to salivary glands. Clin Cancer Res 2006;12:1804-1812. 